\title{
Identification of non-essential loci within the Meleagrid herpesvirus 1 genome
}

\author{
Robyn N. Hall ${ }^{1,2,3,5}$, Joanne Meers ${ }^{1}$, Elizabeth V. Fowler ${ }^{4}$ and Timothy J. Mahony ${ }^{5^{*}}$ (D)
}

\begin{abstract}
Background: Meleagrid herpesvirus 1 (MeHV-1) infectious bacterial artificial chromosomes (iBACs) are ideal vectors for the development of recombinant vaccines for the poultry industry. However, the full potential of iBACS as vectors can only be realised after thorough genetic characterisation, including identification of those genetic locations that are non-essential for virus replication. Generally, transposition has proven to be a highly effective strategy for rapid and efficient mutagenesis of iBAC clones. The current study describes the characterisation of 34 MeHV-1 mutants containing transposon insertions within the pMeHV1-C18 iBAC genome.

Methods: Tn5 and MuA transposition methods were used to generate a library of 76 MeHV-1 insertion mutants. The capacity of each mutant to facilitate the recovery of infectious MeHV-1 was determined by the transfection of clone DNA into chicken embryo fibroblasts.

Results: Attempts to recover infectious virus from the modified clones identified 14 genetic locations that were essential for MeHV-1 replication in cell culture. Infectious MeHV-1 was recovered from the remaining 14 intragenic insertion mutants and six intergenic insertion mutants, suggesting that the respective insertion locations are non-essential for MeHV-1 replication in cell culture.

Conclusions: The essential and non-essential designations for those MeHV-1 genes characterised in this study were generally in agreement with previous reports for other herpesviruses homologues. However, the requirement for the mardivirus-specific genes LORF4A and LORF5 are reported for the first time. These findings will help direct future work on the development of recombinant poultry vaccines using MeHV-1 as a vector by identifying potential transgene insertion sites within the viral genome.
\end{abstract}

\section{Background}

Meleagrid herpesvirus 1 (MeHV-1), commonly known as turkey herpesvirus (HVT), is a non-pathogenic avian herpesvirus originally isolated from turkeys in 1969 [1, 2]. The virus is assigned to the genus Mardivirus, which also includes oncogenic Gallid herpesvirus 2 (GaHV-2), the causative agent of Marek's disease (MD), and the nononcogenic Gallid herpesvirus 3 (GaHV-3). Marek's disease is a highly contagious neoplastic poultry disease of major economic significance worldwide. The close antigenic relationship amongst the mardiviruses has been exploited since the 1970s through the use of MeHV-1 as a live vaccine to reduce production losses resulting from MD [3]. However, despite widespread vaccination with either

\footnotetext{
*Correspondence: t.mahony@uq.edu.au

${ }^{5}$ Queensland Alliance for Agriculture and Food Innovation, Centre for Animal Science, The University of Queensland, Brisbane, QLD 4072, Australia Full list of author information is available at the end of the article
}

MeHV-1, bivalent vaccines containing MeHV-1/GaHV-3 or attenuated GaHV-2 strains, MD outbreaks continue to occur. The isolation of GaHV-2 field strains of increased virulence has been correlated with the loss of protective capacity of these vaccines, which reinforces the need for development of improved MD vaccines [4,5]. It is likely that novel vaccines targeting GaHV-2 will be constructed using recombinant DNA technologies, for which MeHV-1 is ideally suited as a vector candidate.

In addition to its use as a vaccine against MD, MeHV1 is also widely utilised as a recombinant vaccine vector for poultry diseases such as infectious laryngotracheitis, Newcastle disease, infectious bursal disease and highly pathogenic avian influenza [6-9]. Currently, only a limited number of transgene insertion sites are used in the development of recombinant MeHV-1 based vaccines. Use of a suboptimal insertion site can have a pronounced effect on vaccine efficacy. For example, Gao 
et al. [10] reported a reduced post-challenge mortality with highly pathogenic avian influenza virus when the haemagglutinin gene was expressed from the MeHV-1 US2 locus compared to the US10 locus, likely because in vivo virus replication was more affected with the disruption of US1O compared to US2. Thus the identification of alternative transgene insertion sites will be useful for the optimisation of MeHV-1 as a vaccine vector.

The MeHV-1 genome is $159,160 \mathrm{bp}$ in length and has a type 4 herpesvirus genomic structure [11, 12]. It comprises a unique long $\left(\mathrm{U}_{\mathrm{L}}\right)$ and a unique short $\left(\mathrm{U}_{\mathrm{S}}\right)$ region, flanked by terminal/internal repeat long $\left(\mathrm{TR}_{\mathrm{L}} / \mathrm{IR}_{\mathrm{L}}\right)$ and short segments $\left(\mathrm{TR}_{\mathrm{S}} / \mathrm{IR}_{\mathrm{S}}\right)$, respectively $[11,12]$. In addition, the genomic termini comprise telomeric repeats, or a-like sequences, of variable length, which are cis-acting elements involved in genome packaging [13]. These a-like sequences are also present at the $\mathrm{IR}_{\mathrm{L}} / \mathrm{IR}_{\mathrm{S}}$ junction [11]. For comparative purposes, this article refers to individual genes by their putative human herpesvirus 1 (HHV-1) homologue, using $\mathrm{U}_{\mathrm{L}}$ and $\mathrm{U}_{\mathrm{S}}$ nomenclature [14]. Genes unique to mardiviruses are identified by their protein designation, as described in the MeHV-1 reference sequence [Genbank: NC_002641.1] [11]. The complete MeHV-1 genome encodes 79 putative genes [12]. Of these, 73 are single copy; 66 within the $U_{L}$ region and seven in the $U_{S}$ region. The genes $v N R 13$ and icp 4 are duplicated, with one copy of each located in the $\mathrm{IR}_{\mathrm{S}}$ and $T R_{\mathrm{S}}$ elements. The US8 gene, encoding the envelope glycoprotein $\mathrm{E}(\mathrm{gE})$, spans the $\mathrm{TR}_{\mathrm{S}}-\mathrm{U}_{\mathrm{S}}$ boundary region; consequently, the gene US8\%, located within the $I R_{S}$, is a truncated duplication of US8. Current knowledge of MeHV-1 gene function has largely been extrapolated from studies on GaHV-2, and more broadly from genetic studies of HHV-1 and other herpesviruses. While MeHV-1 is currently utilised as a vaccine vector, a more detailed understanding of the genetic background of this virus is required to facilitate its further development as a vector.

The establishment of infectious bacterial artificial chromosome (iBAC) technologies for herpesvirus mutagenesis has simplified the process of generating modified viruses for functional studies and recombinant vaccine construction. For global genome mutagenesis studies, transposition has previously been proven to be a valuable tool, since the random insertion of transposon sequences allows for the efficient generation of a library of unique insertional mutants. These mutants can then be screened to determine if the transposon insertion affects the replication capacity of the virus in cell culture [15-19]. In this way, non-essential genetic loci can be readily identified, and concurrently tested for their potential to carry transgenes for the subsequent generation of recombinant vaccines.

The aim of this study was to characterise a MeHV-1 iBAC transposition library by determining the site of transposon insertion and the impact on viral replication in cell culture. Overall, twenty non-essential loci were identified within the MeHV-1 genome. Additionally, the requirement for the mardivirus-specific genes LORF4A and $L O R F 5$ are reported for the first time.

\section{Results}

\section{Transposition into a MeHV-1 BAC}

The MeHV-1 iBAC clone used in this study was pMeHV1C18. It has recently been reported that pMeHV1-C18 has an in vitro replication capacity similar to wild-type MeHV1 despite lacking functional copies of seven genes [20]. The construction of a combined Tn5 and MuA transposition library of pMeHV1-C18 has been described previously [21]. Two transposition systems, Tn5 and MuA, were utilised during the generation of this library due to the early finding that the MeHV-1 genome is partially resistant to Tn5 transposition. The MuA transposon construct was engineered to contain an eGFP marker gene. Due to the observed resistance to $\operatorname{Tn} 5$ transposition, only a minimal $\operatorname{Tn} 5$ transposon construct was successfully utilised for Tn5 transposition. Briefly, the final library contained 76 mutants with insertions mapping to the MeHV-1 genome, disrupting 30 intragenic and six intergenic locations (Table 1; Additional file 1: Supplemental Table S1). Constructs were screened by restriction enzyme analysis and by bi-directional Sanger sequencing outwards from the 5 ' and 3 ' termini of the transposon insertion [21]. Results from these screening analyses were consistent with a single insertion event occurring within each construct.

Forty-seven insertion events mapped to within the $U_{L}$ genomic region, three events mapped to the $U_{S}$ region, two insertions were identified within the $\mathrm{IR}_{\mathrm{L}} / \mathrm{TR}_{\mathrm{L}}$ regions and 22 insertions mapped to the $\mathrm{IR}_{\mathrm{S}} / \mathrm{TR}_{\mathrm{S}}$ regions. Additionally, two insertions mapped to the a-like sequences. Recovery of virus from transposed clones was assessed by observing characteristic MeHV-1 cytopathic effect (CPE) using light microscopy, and whenever possible, by detecting the expression of enhanced green fluorescent protein (eGFP) using fluorescent microscopy (the MuA transposon used to generate $\mathrm{MuA} \triangle 48-84$ contained an eGFP transgene) (Fig. 1). Cell monolayers were passaged at least once after CPE was evident to confirm the presence of infectious virus. Where CPE was not observed, monolayers were blind passaged three to four times to confirm the absence of infectious virus.

\section{Transposition into the $U_{L}$ region}

Within the $\mathrm{U}_{\mathrm{L}}$ genomic region, 25 genes were disrupted by transposon insertion events. Of these, 13 and 12 locations were found to be essential and non-essential for replication of MeHV-1 in cell culture respectively (Table 1).

Although UL13 and UL53 transposition mutants were classed as non-essential based on the observation of 
Table 1 Summary of transposon-mediated gene interruptions within the coding regions of the MeHV-1 infectious clone pMeHV1-C18. The genes affected and encoded gene products are shown

\begin{tabular}{|c|c|c|c|c|c|c|c|}
\hline Gene/element & Gene product/function & pMeHV1-C18 & GaHV-21 & $\mathrm{HHV}-1^{2}$ & $\mathrm{HHV}-3^{3}$ & SuHV-1 ${ }^{4}$ & $\mathrm{BoHV}-1^{5}$ \\
\hline VLip & Viral lipase & $\mathrm{NE}$ & $\mathrm{NE}$ & NA & NA & NA & NA \\
\hline LORF2 & Unknown & $\mathrm{NE}$ & $\mathrm{E} / \mathrm{NE}$ & NA & NA & NA & NA \\
\hline UL6 & Portal protein & E & ND & E & E & ND & E \\
\hline UL8 & Helicase/ primase associated protein & E & ND & E & E & ND & E \\
\hline UL9 & Origin binding protein & E & ND & E & $\mathrm{E}$ & $\mathrm{E}$ & $\mathrm{E}$ \\
\hline UL10 & Glycoprotein M & $\mathrm{NE}$ & $\mathrm{E}$ & NE & E & E & $\mathrm{NE}$ \\
\hline UL13 & Protein kinase & A & $\mathrm{NE}$ & NE & $\mathrm{NE}$ & $\mathrm{NE}$ & $\mathrm{NE}$ \\
\hline UL17 & Tegument/DNA packaging protein & E & $\mathrm{E}$ & E & E & $\mathrm{E}$ & E \\
\hline UL19 & VP5 capsid protein & E & $\mathrm{E}$ & E & E & $\mathrm{NE}$ & $\mathrm{E}$ \\
\hline UL21 & Tegument protein & A & ND & $\mathrm{NE}$ & E & $\mathrm{NE}$ & $\mathrm{NE}$ \\
\hline UL26 & Scaffold protease & E & ND & E & E & ND & E \\
\hline UL26.5 & Scaffold protein & E & ND & E & E & ND & E \\
\hline UL27 & Glycoprotein B & $E^{*}$ & $\mathrm{E}$ & E & E & ND & E \\
\hline UL29 & Single stranded DNA binding protein & E & ND & E & E & $\mathrm{E}$ & $\mathrm{E}$ \\
\hline UL36 & Large tegument protein & E & $\mathrm{E}$ & E & E & E & E \\
\hline UL37 & Tegument protein & $\mathrm{E}$ & $\mathrm{E}$ & $\mathrm{E}$ & E & ND & $\mathrm{E}$ \\
\hline UL39 & Ribonucleotide reductase large subunit & $\mathrm{NE}$ & $\mathrm{NE}$ & NE & $\mathrm{NE}$ & $\mathrm{NE}$ & $\mathrm{NE}$ \\
\hline UL40 & Ribonucleotide reductase small subunit & $N E^{*}$ & ND & NE & NE & ND & $\mathrm{NE}$ \\
\hline UL42 & DNA polymerase processivity subfactor & $E$ & ND & E & E & ND & E \\
\hline UL47 & VP13/14 capsid protein & $\mathrm{NE}$ & $\mathrm{NE}$ & NE & $\mathrm{NE}$ & $\mathrm{NE}$ & $\mathrm{NE}$ \\
\hline UL48 & VP16 a-transinducing factor & NE & $\mathrm{NE}$ & E & $\mathrm{NE}$ & $\mathrm{NE}$ & $\mathrm{NE}$ \\
\hline UL52 & Helicase/ primase associated protein & $\mathrm{E}$ & ND & $\mathrm{E}$ & E & ND & $\mathrm{E}$ \\
\hline UL53 & Glycoprotein K & A & $\mathrm{E}$ & $\mathrm{NE}$ & $\mathrm{E}$ & $\mathrm{E}$ & $\mathrm{E}$ \\
\hline LORFAA & Unknown & NE & ND & NA & NA & NA & NA \\
\hline LORF5 & Unknown & NE\# & $\mathrm{NE}$ & NA & NA & NA & NA \\
\hline$i c p 4$ & Major immediate early regulatory gene & $E$ & ND & E & E & ND & ND \\
\hline US3 & Protein kinase & $\mathrm{NE}$ & $\mathrm{NE}$ & NE & E & $\mathrm{NE}$ & $\mathrm{NE}$ \\
\hline US6 & Glycoprotein D & $N E^{*}$ & $\mathrm{NE}$ & E & ND & $\mathrm{NE}$ & $\mathrm{E}$ \\
\hline
\end{tabular}

The gene requirements for pMeHV1-C18 determined in this study are designated as either essential (E), non-essential (NE) or severely attenuated (A). Comparative data, using the same nomenclature, is shown for selected alphaherpesviruses; Gallid herpesvirus 2 (GaHV-2), Human herpesvirus 1 (HHV-1), Human herpesvirus 3 (HHV-3), Suid herpesvirus 1 (SuHV-1) and Bovine herpesvirus 1 (BoHV-1). Not applicable (NA) denotes genes unique to the genus Mardivirus. Rows in bold highlight those genes for which requirement varies between the viruses listed. *Requirement for growth in cell culture previously reported in wildtype MeHV-1 virus [57-59]

\#Transposon was unstable in viral genome

References for gene interruption studies: ${ }^{1}[18,23,25,42,49,57,60-67] ;{ }^{2}[14,35,38,58,68-76] ;{ }^{3}[26,41,45,77,78] ;{ }^{4}[17,27,43,48,79-82] ;{ }^{5}[19,83]$

persistent eGFP expression after multiple passages, the recovered viruses from these clones showed severely attenuated replication in cell culture compared to the parental virus (Fig.1e and 1f, Table 1).

The UL21 disruption mutants, Tn5 $\Delta 14, M u A \Delta 37$ and MuA $\triangle 41$, also caused a very low grade CPE compared to the parental virus. As these constructs were generated with transposons lacking the eGFP reporter gene, expression of eGFP could not be used to verify virus replication in these clones. Instead, these clones were confirmed to facilitate the recovery of infectious MeHV-1 through detection of viral DNA in the cell monolayer using PCR after three or more passages. To exclude the possibility that the PCR assay was amplifying residual transfected iBAC DNA, the pMeHV1-C18-MuA $\Delta 64$ construct, a non-infectious glycoprotein B disruption mutant (Fig. $1 \mathrm{~g}$ and $1 \mathrm{~h}$ ), was assayed in parallel with the same PCR assay. As expected, no pMeHV1-C18-MuA $\Delta 64$ DNA was detectable at passage three, confirming that DNA detected from UL21 disruption mutants was due to ongoing viral replication. Based on the continued detection of viral DNA, the UL21 insertion site was designated as nonessential for MeHV-1 replication in cell culture, although replication was markedly impaired. 


\section{Wild-type}

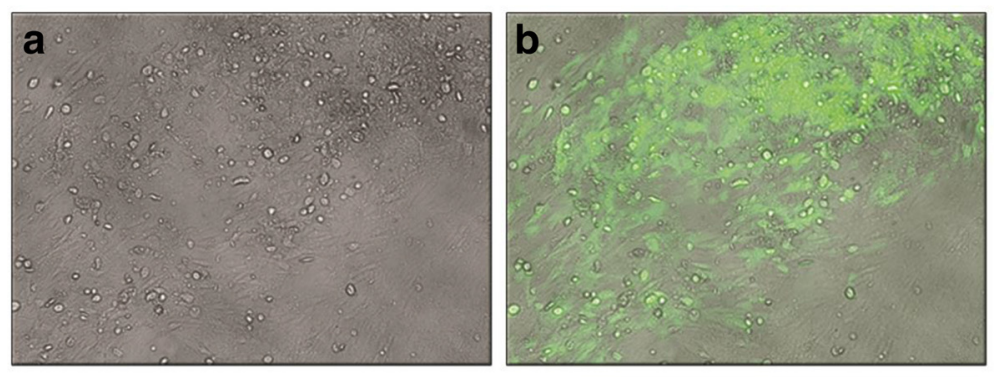

Non-essential, no attenuation

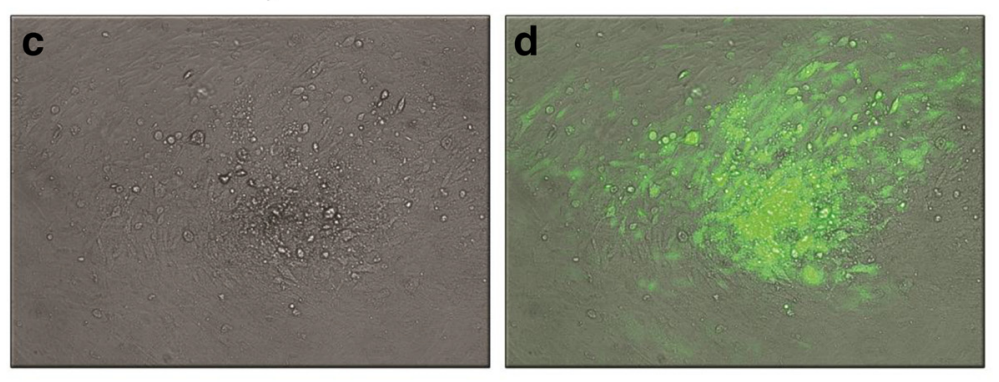

Non-essential, severe attenuation

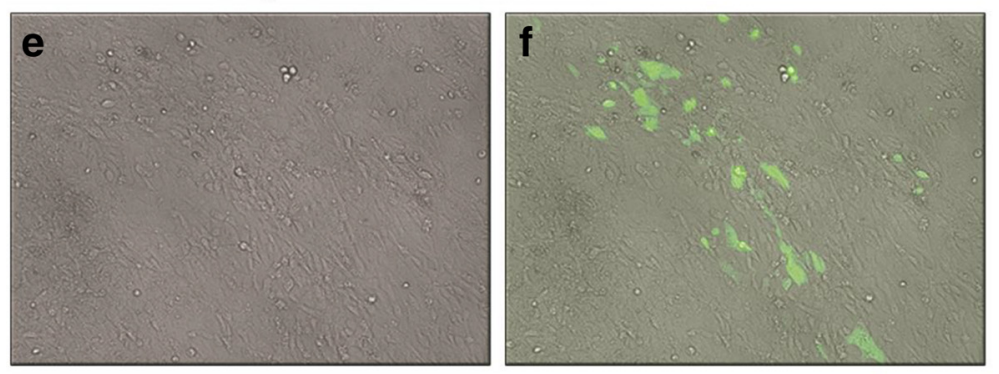

\section{Essential, complete attenuation}
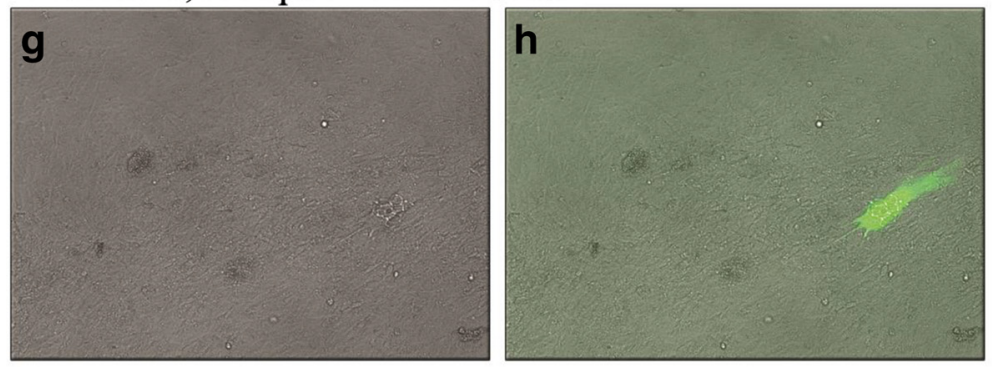

Fig. 1 Classification of the replication capacity of transposition mutants of MeHV-1 in cell culture. Gene requirements of MeHV-1 were assigned based on the replication capacity of the respective transposition mutants in cell culture when compared to parental virus (MuA $\Delta 65$ day 5 post-transfection a: brightfield $\times 100$; and $\mathbf{b}$ : fluorescent microscopy $\times 100$ ). Clones were classified as 'non-essential, no attenuation' (MuA $\Delta 72$ day 5 post-transfection c: brightfield $\times 100$; and $\mathbf{d}$ : fluorescent microscopy $\times 100$ ), 'non-essential, severe attenuation' (MuA $\Delta 68$ day 7 post-transfection e: brightfield $\times 100$; and $\mathbf{f}$ : fluorescent microscopy $\times 100$ ), or 'essential, complete attenuation' (MuA $\Delta 64$ day 7 post-transfection $\mathbf{g}$ : brightfield $\times 100$; and $\mathbf{h}$ : fluorescent microscopy $\times 100$ ). BAC DNA encoding the MeHV-1 genome and containing a single transposon insertion within either the BAC vector backbone, therefore reflecting parental virus ( $\mathbf{a}$ and $\mathbf{b}$ ), UL48 (c and $\mathbf{d}), U L 53$ (e and $\mathbf{f}$ ) or UL27 (g and $\mathbf{h}$ ) was transfected into CEFs. Monolayers were passaged every five to eight days and were observed for the development of CPE using light microscopy (a, $\mathbf{c}, \mathbf{e}$ and $\mathbf{g})$ and, whenever possible, for expression of a marker gene (eGFP) using fluorescent microscopy (b, $\mathbf{d}, \mathbf{f}$ and $\mathbf{h}$ )

The LORF5 gene was found to be non-essential for viral replication in cell culture, since CPE was observed for the disruption mutants pMeHV1-C18-MuA $\Delta 59$ and $\mathrm{MuA} \Delta 82$. However, both mutants were observed to partially lose eGFP expression by passage three in cell culture, based on the presence of both eGFP 
expressing foci of CPE as expected, as well as noneGFP expressing foci.

\section{Transposition into the $\mathrm{U}_{\mathrm{S}}$ region}

Two genes within the $U_{S}$ region, US3 and US6, were disrupted by transposon insertion events. Both insertion locations were determined to be non-essential for MeHV-1 replication in cell culture (Table 1).

\section{Transposition into the $\mathbf{I R}_{\mathbf{S}} / \mathrm{TR}_{\mathbf{S}}$}

As previously reported, the $\mathrm{IR}_{\mathrm{S}} / \mathrm{TR}_{\mathrm{S}}$ of MeHV-1 were transposed at higher frequency compared to the rest of the viral genome [21]. Of the 76 pMeHV1-C18 transposition mutants generated, $22(29 \%)$ mapped to the $\mathrm{IR}_{\mathrm{S}} / \mathrm{TR}_{\mathrm{S}}$ regions of the genome. Eleven of these insertions were within the icp 4 coding region. Three clones, pMeHV1$\mathrm{C} 18-\operatorname{Tn} 5 \Delta 13, \operatorname{Tn} 5 \Delta 21$ and MuA $\Delta 76$, mapped within the repeated segment of the US8 gene. The transposition events in seven clones were mapped to non-coding sequences of the $\mathrm{IR}_{\mathrm{S}} / \mathrm{TR} \mathrm{R}_{\mathrm{S}}$ elements. The remaining clone, pMeHV1-C18-MuA $\Delta 46$, contained a transposon insertion within the dual-copy gene $v N R 13$.
All insertions mapped to different nucleotide positions within the $I R_{S} / T R_{S}$ elements, demonstrating that these clones originated from independent transposition events (Additional file 1: Supplemental Table S1). Insertion events generated using the $\operatorname{Tn} 5<\mathrm{KAN}-2>$ transposon were mapped to a specific repeat based on the presence of suitable restriction endonuclease (RE) cleavage sites within both the transposon construct and the iBAC (Fig. 2). Interestingly, of the 10 insertions generated by $\mathrm{Tn} 5$ transposition that mapped to the $\mathrm{IR}_{\mathrm{S}} / \mathrm{TR}_{\mathrm{S}}$ regions, eight were within the $\mathrm{TR}_{\mathrm{S}}$. Virus was successfully recovered from all $\operatorname{Tn} 5$ transposon mutants with insertions in the $\mathrm{IR}_{\mathrm{S}} / \mathrm{TR}_{\mathrm{S}}$. However, this did not reflect the requirement of the genetic elements at the location of the insertion for viral replication, since the untransposed copy of the gene may compensate for loss of the transposed copy. Furthermore, it was anticipated that recombination between the $I R_{S}$ and $T R_{S}$ elements during viral replication could result in the recovery of the parental iBAC genotype (Fig. 2).

In an attempt to determine the requirement of dualcopy genes, naturally occurring recombination between a
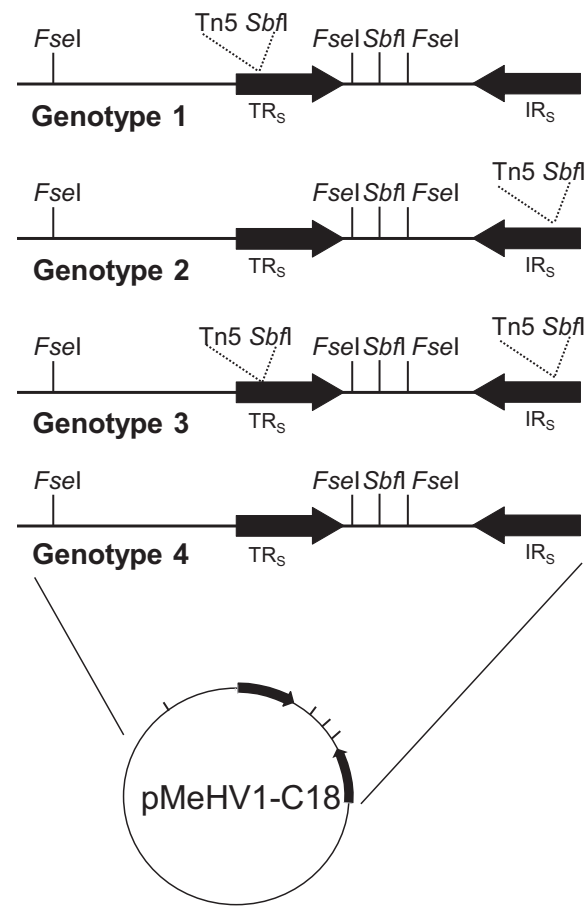

b

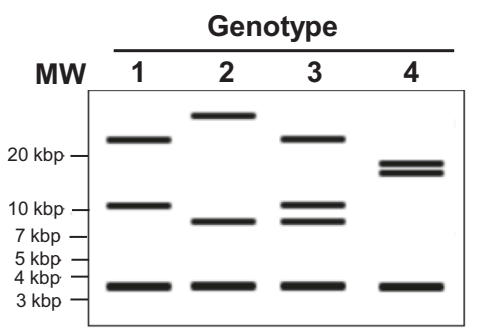

c Genotype

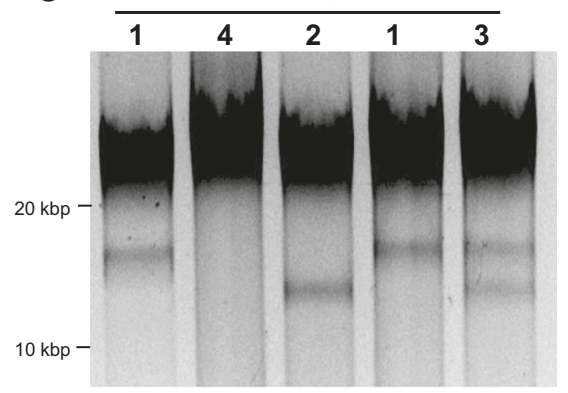

Fig. 2 Restriction fragment length polymorphism of icp4 insertion mutants a Schematic representation of the four potential genotypes resulting from recombination between the $\mathrm{IR}_{S} / \mathrm{TR}_{\mathrm{S}}$ elements of pMeHV1-C18-Tn5 $\Delta$-1 during virus replication. Genotype 1: Transposon in TR Inversion of transposon to the IRs; Genotype 3: Duplication of transposon; Genotype 4: Loss of transposon. (b) In silico Fsel/Sbfl restriction endonuclease digestion patterns for pMeHV1-C18-Tn5 $\Delta 1$ Genotypes 1, 2, 3, and 4. (c) iBAC DNA co-digested with Fsel and Sbfl. lane 1: pMeHV1-C18-Tn5 1 (genotype 1); lane 2: loss of transposon (genotype 4); lane 3: transposon in IRS element (genotype 2); lane 4: transposon in $\mathrm{TR}_{\mathrm{S}}$ element (genotype 1); lane 5: duplication of transposon (genotype 3) 
the $I R_{S} / T_{S}$ elements during viral replication was exploited to isolate double-insertional mutants [22]. This was investigated using the icp4 disruption mutant, pMeHV1-C18-Tn5 $\Delta 1$. It was postulated that following recovery of infectious MeHV-1 and subsequent viral replication, recombination between the $\mathrm{IR}_{\mathrm{S}}$ and $T R_{\mathrm{S}}$ regions during replication would result in the generation of four genotypes as illustrated in Fig.2a. These genotypes were: genotype 1, a single transposon insertion in the $T R_{S}$ as described for pMeHV1-C18-Tn $5 \Delta 1$; genotype 2 , characterised by an inversion of the repeat sequence elements, transferring the original transposon insertion to the $I_{S}$ element; genotype 3 , replacement of the unmodified $I_{S}$ with the transposed $\mathrm{TR}_{\mathrm{S}}$ sequence caused a duplication of the $\mathrm{TR}_{\mathrm{S}}$ transposon element, thus generating a double icp4 disruption mutant; or genotype 4, a duplication of the $\mathrm{IR}_{\mathrm{S}}$ element eliminating the transposed $\mathrm{TR}_{\mathrm{S}}$ element, thereby restoring the parental iBAC sequence. The requirement of icp 4 for replication can be assessed using a genotype 3 construct, because of its icp4-negative genotype (Fig.2a).

To determine which of these genotypes could be recovered from cells infected with virus recovered from pMeHV1-C18-Tn5 $\Delta 1$, total DNA was isolated and electroporated into bacterial cells. Putative iBAC DNA were recovered from chloramphenicol resistant bacteria and digested with FseI and Sbfl. In silico analyses suggested that double-digestion of Tn 5 transposition mutants with these restriction enzymes would generate fragment profiles characteristic for the genotypes described previously (Fig.2b). All four genotypes could be distinguished by the presence or absence of two polymorphic fragments with a predicted size of $\sim 12 \mathrm{kbp}$ and $\sim 8.5 \mathrm{kbp}$.

The RE patterns of five recovered pMeHV1-C18-Tn $5 \Delta 1$ iBAC clones digested with FseI and Sbfl are shown in Fig.2c. The predicted polymorphic fragments generated by the addition of the $S b f l$ site by $\mathrm{Tn} 5$ transposition into the $\mathrm{TR}_{S} / \mathrm{IR}_{\mathrm{S}}$ elements were clearly distinguishable, although their estimated sizes were larger than expected $(\sim 14.1 \mathrm{kbp}$ and $\sim 12.5 \mathrm{kbp}$; Fig.2c). There are several factors that may have retarded the migration of these fragments including overloading of iBAC DNA, presence of impurities or excess bacterial host DNA. All potential genotypes generated two large fragments, represented by the large fluorescent bands (Fig.2b and 2c), which are not individually resolvable by standard agarose gel electrophoresis. The predicted $3.7-\mathrm{kbp}$ and $3.6-\mathrm{kbp}$ fragments were not observed in the electrophoretic analyses due to their relatively small size; thus these fragments represented only a very small proportion of the total DNA and their staining was expected to be beyond the limits of detection. As these fragments were identical in all potential genotypes and therefore visualisation of these fragments was not necessary for evaluation of the assay.
Recovered clones that had the larger polymorphic fragment were classified as genotype 1 , while clones with the smaller polymorphic fragment were identified as genotype 2 . Clones with both fragments were designated as icp4-negative mutants (genotype 3). Clones with neither of these fragments had reverted to the genotype of the parental iBAC (genotype 4). Of the 41 clones subjected to digestion with Sbfl and FseI, 10 clones were classified as genotype 1, 11 clones were genotype 2, five clones were determined to be of genotype 3 and 10 clones were genotype 4 . The RE fragment profiles of the remaining five clones analysed were inconsistent with the parental clone, pMeHV1-C18-Tn5 $\Delta 1$, and were discarded from further analyses.

One dual-icp4 disruption clone (genotype 3), pMeHV1-C18-Tn5 $\Delta 1-3$, was used to investigate the requirement for icp4 for MeHV-1 replication in cell culture. Transfection of this construct into chicken embryo fibroblasts (CEFs) did not result in any observable CPE after three passages, demonstrating that $i c p 4$ is essential for MeHV-1 replication in cell culture. Although the approach described above resulted in the isolation of a dual-copy icp4 disruption mutant, it was not successfully applied to the isolation of dual-copy mutants for other genes in $\mathrm{IR}_{\mathrm{S}} / \mathrm{TR} \mathrm{R}_{\mathrm{S}}$ regions.

\section{Transposition into the $I R_{L} / T_{L}$}

Two MuA transposon insertion events mapped to the noncoding sequences of the $\mathrm{IR}_{\mathrm{L}} / \mathrm{TR}_{\mathrm{L}}$ regions. Since insertions events were isolated from $\mathrm{MuA}$ transposition reactions, they could not be localised to individual repeats due to lack of suitable RE sites in the transposon construct, and dualcopy disruption mutants could not be not generated.

\section{Transposition into a-like sequences}

Two transposed clones, pMeHV1-C18-Tn5 16 and $\operatorname{Tn} 5 \Delta 20$, contained insertions within the a-like sequences of the MeHV-1 genome (Additional file 1: Supplemental Table S1). The exact nucleotide position of the insertion could not be determined for these clones, since the nucleotide sequence data did not extend beyond the termini of this repetitive sequence element. Transfection of $\operatorname{Tn} 5 \Delta 16$, with a single transposon insertion into an a-like sequence, produced CPE indistinguishable from that of wild-type MeHV-1. However, because only one copy of the a-like sequences was disrupted, the requirement of these regions for replication of MeHV-1 in cell culture could not be determined in the current study.

\section{Discussion}

The requirements for 28 coding and six non-coding regions within the MeHV-1 genome have been determined in this study, using a library of 76 transposition mutants. These included 11 genes for which the in vitro requirements for 
replication have not previously been reported in any of the mardiviruses (Table 1). Clones with unique phenotypes and gene designations that contrast with the reported literature are discussed in detail below. Of particular interest in this study were those insertion sites that were non-essential for replication, as these represent potential transgene insertion sites for MeHV-1-based vectors. These sites included 14 intragenic and six intergenic sites.

Homologues of the LORF2 gene are restricted to the Mardivirus genus. It has been suggested that this gene may have a role in mRNA transcription or processing but this is yet to be experimentally confirmed [12]. Recently, the GaHV-2 LORF2 homologue was reported to have immunoevasion functions via the downregulation of MHC class I in infected cells [23]. LORF2 has previously been reported as essential for GaHV-2 replication in cell culture [18]. In contrast, another study reported retroviral insertions within the ORF as having no effect on GaHV-2 replication [23, 24]. In the current study, transposition clone MuA $\triangle 30$ contained an insertion within the first exon of MeHV-1 LORF2, disrupting $99.5 \%$ of the predicted polypeptide. Viral recovery experiments clearly demonstrated that this location was non-essential for replication of MeHV-1 in cell culture, with CPE developing within five days of transfection. The differing requirement of this gene in MeHV-1 and GaHV2 is of interest, and further investigations into LORF2 function in MeHV-1 are warranted. Furthermore, the use of LORF2 as a transgene insertion site for vaccine development may increase vaccine efficacy through impairment of the proposed LORF2 immunoevasion functions.

The non-essential classification of MeHV-1 UL1O in this study contrasts with the essential assignment of the GaHV-2 UL10 homologue (Table 1) [25]. The UL10 gene encodes a homologue of glycoprotein $\mathrm{M}(\mathrm{gM})$, a core herpesvirus gene $[11,12]$. The $U L 10$ gene is essential in the strictly cell-associated viruses GaHV-2 and Human herpesvirus 3 (HHV-3) [25, 26]. In contrast, UL10 has consistently been reported as non-essential for viral replication in cell culture for cell-free herpesviruses such as HHV-1, Suid herpesvirus 1 (SuHV-1), Bovine herpesvirus 1 (BoHV-1), Equine herpesvirus 1 (EHV-1) and Gallid herpesvirus 1 [19, 27-30]. Although the parental MeHV-1 strain FC126 used in this study was cell-associated, cell-free virus is produced to a limited extent and this strain can be adapted to produce high titres of cell-free virus [31]. It has also been suggested that expression of glycoprotein $\mathrm{D}(\mathrm{gD})$ may compensate for loss of gM function, since both HHV-3 and GaHV-2 do not express gD in cell culture, and this may explain the essential designation of $\mathrm{gM}$ in these viruses [25]. While the expression of MeHV-1 gD during infection in cell culture has not been reported to date, the capacity of the virus to adapt to cell-free growth suggests it is.
The UL21 gene encodes a poorly characterised tegument protein that is capsid-associated and may have roles in intracellular transport and in nuclear egress $[32,33]$. The recovery of infectious MeHV-1 from three $U L 21$ transposed clones, $\operatorname{Tn} 5 \Delta 14, \mathrm{MuA} \Delta 37$ and $\operatorname{MuA} \Delta 41$, in combination with the presence of viral DNA after sequential passages, confirms that MeHV-1 UL21 is nonessential for replication in cell culture. However, replication was severely attenuated compared to the parent virus. Disruption studies in other alphaherpesviruses have shown UL21 to be non-essential, although a range of deleterious effects have been noted on virus replication (Table 1). A UL21 mutant of SuHV-1 showed impaired replication in cell culture and reduced virulence in vivo $[17,34]$. For HHV-1 and BoHV-1, UL21 has been shown to be non-essential, but deletion reduced the in vivo replication capacity of HHV-1 [19, 35, 36]. In contrast, UL21 has been reported to be essential for Human herpesvirus 2, HHV-3 and EHV-1 [26, 32, 37]. The severe attenuation observed for MeHV-1 in this study suggests the $U L 21$ gene is unsuitable for use in recombinant vaccine applications, however it may be of use for generating replication-limited gene delivery constructs for poultry research applications.

The non-essential phenotype of MeHV-1 UL48 disruption mutants characterised in this study conflicts with the essential requirement of this gene for HHV-1 replication (Fig.1c and 1d). In HHV-1, UL48 encodes the VP16 $\alpha$-trans inducing factor, a tegument protein that induces immediate-early gene transcription and is also required for virion assembly [38]. The UL48 homologues of many alphaherpesviruses, including mardiviruses, lack the acidic carboxyl terminus transactivating domain present in the HHV-1 UL48 protein, however transactivating functions may be retained via other transactivation sites within UL48 $[11,12,39,40]$. This gene is essential for the replication of HHV-1 and EHV-1 in cell culture, but is nonessential in other alphaherpesviruses investigated to date, including HHV-3, SuHV-1, BoHV-1 and GaHV-2 (Table 1) $[19,26,38,41-45]$.

The MeHV-1 UL53 gene is a homologue of the HHV-1 gene encoding glycoprotein $\mathrm{K}(\mathrm{gK})[11,12]$. Similar to other viral glycoproteins, $\mathrm{gK}$ has roles in cell-to-cell fusion and in viral egress from infected cells [46, 47]. It has been reported to be essential for replication of many alphaherpesviruses, including GaHV-2, HHV-3, SuHV-1 and BoHV-1 (Table 1) [19, 26, 48, 49], while it is non-essential for HHV-1 and EHV-1 growth in vivo [50, 51]. Interestingly, deletion of UL53 from both the HHV-1 and EHV-1 genomes resulted in severely attenuated viruses with greatly reduced plaque sizes and impaired virion penetration in cell culture [37, 51, 52]. Marked attenuation was also observed for the MeHV-1 UL53 disruption mutant, $\mathrm{MuA} \Delta 68$, in this study (Fig.1e and 1f). 
The MeHV-1 LORF4A gene is a homologue of LORF4 genes of GaHV-2 and GaHV-3 and LORF9 of Anatid herpesvirus 1, and the encoded polypeptide shares $47 \%$ amino acid identity to the proposed paralogue, MeHV-1 LORF4 [11, 12]. The LORF4 homologues have been postulated to have roles as avian host range determinants, since the occurrence of this gene is restricted to mardiviruses [11, 12]. Transposon insertion into LORF4A in the pMeHV1-C18 transposon clone Tn5 10 disrupted $82 \%$ of the gene, and the insertion location was designated as non-essential for virus growth in cell culture. This is the first report of a disruption mutant of LORF4.

Overall fourteen genetic locations were identified as essential for MeHV-1 replication in cell culture (Table 1). The classification of these loci provides additional foundational information concerning MeHV-1 replication, as the requirements of 13 of these genes have not previously been reported for MeHV-1. Although it would have been interesting to determine the effects of insertions on global viral gene expression and protein production, this was beyond the scope of the current study. Similarly, revertant constructs were not generated for replication-defective mutants since putatively essential genes are not of further interest for vaccine development.

It is noteworthy that the transposition mutants reported here are cumulative gene deletion mutants of MeHV-1, as pMeHV1-C18 lacks seven coding regions compared to the parental virus [20]. This genetic background may have contributed to the observed attenuation of some clones compared to the wild-type MeHV-1. It is considered unlikely that the requirement of the non-essential loci identified in this study would be essential in the fulllength virus, as it is reasonable to conclude that effects on viral replication are likely to be more severe with cumulative gene deletions compared to the disruption of a single gene. However, it is possible the locations designated as essential in this study may be non-essential in the parental virus. Nonetheless, this is also considered unlikely as the MeHV-1 genes designated as essential in this study conform with the reported requirements for the respective homologues of other alphaherpesviruses, with the exception of UL19 which is reported as non-essential in SuHV1 (Table 1) [17]. However, it must be noted that in that study, the transposon insertion event mapped 2 bp downstream of the SuHV-1 UL19 ORF, therefore it could be argued that this was not a true report of the UL19 requirement in this virus as complete translation of the encoded polypeptide would have been possible.

Given the instability observed in the LORF5 insertion mutants, it is possible that this is an essential gene and it may have been misclassified as non-essential in the current study. This is considered unlikely, since in the case of the LORF5 mutants, the transgene was gradually lost during serial passage of recovered virus. In the case of an insertion into an essential gene, the insertion mutant would not undergo sufficient replication capacity to facilitate loss of the transgene and subsequent recovery of virus. Regardless of whether the LORF5 gene is essential or non-essential for replication, the observed instability of the transposon insertions in two independent LORF5 transposon insertion mutants suggests this region of the genome would be unsuitable for recombinant vector applications.

Despite the potential limitations of the cumulative gene deletion genotype of the iBAC used in this study, it has enabled the identification of viruses with novel phenotypes, for example the MuA $\Delta 68$ virus with an insertion into UL53. While CPE was observed, it was subtle compared to the parent virus and may have been missed completely in the absence of reporter gene expression (Fig.1e and 1f). It is considered highly unlikely that a virus with this phenotype could be constructed using rational genetargeting strategies.

Importantly, potential insertion sites for vector development must also be verified in vivo, since it is generally accepted that non-essential genes in cell culture may have major roles in vivo, for example in immunoevasion or other virus-host interactions [14]. An example of this are glycoprotein $\mathrm{C}(\mathrm{gC})$-null mutants of GaHV-2, which show increased viral replication in cultured cells, however in vivo infection required a longer incubation period to establish infection, viraemia and induction of seroconversion compared to $\mathrm{gC}$-positive virus, and $\mathrm{gC}$-null viruses were not transmitted horizontally $[53,54]$. The in vivo replication capacity of virus recovered from the parental iBAC used in this study is reduced compared to wild-type MeHV-1 [20]. As a result it might be expected that any constructs derived from this parent clone would be further attenuated in vivo. Extrapolating from the GaHV-2 studies discussed above, a deletion identified in the UL44 region of pMeHV1-C18 likely contributes to the in vivo attenuation observed with this construct. Therefore consideration should be given to the restoration of this deletion prior to in vivo assessment of the non-essential gene mutants constructed in the current study.

The strategy used to determine the replication requirement for icp 4 highlights the power of iBAC technologies, for example to generate a dual-disruption mutant with two mutagenised copies of a repeat element. This strategy was developed after the generation of the transposition libraries, thus the presence of suitable RE sites in the Tn5 transposon and the virus was serendipitous. Future studies investigating genetic elements located in the repeat sequences of herpesvirus iBACs should consider the identification of suitable RE sites within the targeted viral genome to enable the identification of modified specific repeat sequences. If appropriate sites are identified in the 
virus, complementary sites could be readily incorporated into the proposed transgene molecule to facilitate the isolation of double-deletion/disruption mutants.

\section{Conclusions}

Despite the previously reported resistance of the MeHV-1 genome to transposition [21], characterisation of mutant clones obtained using these methods has enabled determination of twenty non-essential genomic locations. When considered together with the parent genotype of the MeHV-1 iBAC used in this study, these results demonstrate the considerable degree of redundancy of genes within the MeHV-1 genome in vitro. Moreover, the genotype of pMeHV1-C18, containing multiple deletions compared to the reference MeHV-1 genome, has enabled the identification of viruses with unique phenotypes, such as the gK and UL21 disruption viruses, which replicated in the virtual absence of CPE. Of interest in future studies would be the sequential restoration of genes into these replication-impaired viruses to determine which genes restore the capacity of recovered virus to cause a $\mathrm{CPE}$ more characteristic of MeHV-1.

\section{Methods}

\section{Transposition libraries}

Construction and characterisation of the MeHV-1 iBAC clone, pMeHV1-C18, is described in Mahony et al. [20]. This iBAC contains cumulative gene deletions compared to the MeHV-1 FC126 strain, along with BAC vector sequences within the SORF3/US2 region, and is genetically defined as pMeHV1-C18DUL43:UL44:UL45:UL56: pp38:SORF3:US2, however the short form (pMeHV1C18) will be retained in this manuscript for simplicity. The generation of pMeHV-C18 transposition clones characterised in this study was reported previously [21].

\section{Restriction endonuclease analyses}

Double digestion of Tn5-transposition mutants was performed with $\mathrm{Sbfl}$ and $F s e \mathrm{I}$ at $37^{\circ} \mathrm{C}$ for $1 \mathrm{~h}$, and reactions were heat-inactivated at $65^{\circ} \mathrm{C}$ for $20 \mathrm{~min}$. Digestion products were resolved at $60 \mathrm{~V}$ for $3 \mathrm{~h}$ in $0.7 \%$ agarose gels in $1 \times$ Tris-acetate-EDTA buffer containing $0.1 \mu \mathrm{g} \mathrm{mL}$ ethidium bromide. Banding patterns were visualised using UV light.

\section{Identification of transposition insertion site and orientation}

To identify transposon insertion location within the parent iBAC, bi-directional sequencing was performed using primers specific for the respective transposons (Additional file 1: Supplemental Table S2). Data were analysed using 4Peaks software (http://www.mekentosj.com/science/4peaks) and mapped to the MeHV-1 genome using Blastn analyses [55].

\section{Growth of pMeHV1-C18 transposition mutants in cell culture}

To assess the capacity of the transposed clones to facilitate the recovery of infectious MeHV-1, DNA was prepared from transposon clones and transfected into CEFs. The CEF cells were maintained in a $5 \% \mathrm{CO}_{2}$ environment at $37{ }^{\circ} \mathrm{C}$ in Medium 199 (Gibco), containing $10 \%$ foetal bovine serum (Gibco) and $1 \times$ Antibiotic-Antimycotic (Gibco). Recovered iBAC DNA was transfected in triplicate into CEFs at $80 \%$ confluency, using Lipofectamine and Plus reagent (Invitrogen) according to manufacturer's instructions. Recovery of virus was assessed on a daily basis for characteristic MeHV-1 CPE using light microscopy, and where possible, by the presence of eGFP expression using fluorescent microscopy. Monolayers were passaged at least once after CPE was evident to confirm the presence of passageable virus. Monolayers in which CPE was not observed, were blind passaged after five to eight days at least three times to confirm the absence of infectious MeHV-1.

\section{Analyses of UL21 disruption mutants}

PCR for detection of UL21 insertion clones was performed on total DNA harvested from infected cultures at the third or sixth passage, as described previously [56]. Total DNA was extracted using the DNeasy blood and tissue kit (Qiagen). Elute $(2 \mu \mathrm{L})$ was used as a template for PCR over the UL21 coding region using oligonucleotide pairs: HVT27Fwd with HVTFrag5Rev, HVT27Fwd with KanMERev, and KanME-Fwd with HVTFrag5Rev (Additional file 1: Supplemental Table S2). Each $50 \mu \mathrm{L}$ PCR reaction contained $1 \times$ PCR buffer - Mg (Invitrogen), $0.2 \mathrm{mM}$ each dNTP, $1.5 \mathrm{mM} \mathrm{MgCl}$, $0.2 \mu \mathrm{M}$ each primer, $1 \mathrm{U}$ Platinum Taq DNA polymerase (Invitrogen) and $2 \mu \mathrm{L}$ template. Cycling conditions were: $94{ }^{\circ} \mathrm{C}$ for $2 \mathrm{~min}$ followed by $30 \mathrm{cy}$ cles of $94{ }^{\circ} \mathrm{C}$ for $30 \mathrm{~s}, 55^{\circ} \mathrm{C}$ for $30 \mathrm{~s}, 72{ }^{\circ} \mathrm{C}$ for 1 min per kbp. PCR products $(5 \mu \mathrm{L})$ were resolved on $1 \%$ agarose gels containing GelRed at $60 \mathrm{~V}$ for 1 to $1.5 \mathrm{~h}$.

\section{Characterisation of transposition events in genomic repeat regions}

BACs were re-isolated from cultures infected with the Tn5 mutant pMeHV1-C18-Tn5 $\Delta 1$, containing a transposon insertion within the $\mathrm{IR}_{\mathrm{S}}$ copy of icp4. Briefly, total DNA was extracted from cultures four days after the third passage, as described previously [56]. Purified DNA (1 $\mu \mathrm{g})$ was electroporated into $50 \mu \mathrm{l}$ of Electromax DH10B E. coli (Invitrogen) and selected for on Luria-Bertani (LB) agar plates containing either $12.5 \mu \mathrm{g} \mathrm{mL} \mathrm{m}^{-1}$ chloramphenicol alone, or with the addition of $30 \mu \mathrm{g} \mathrm{mL}{ }^{-1}$ kanamycin. Antibiotic-resistant colonies containing an iBAC construct were isolated, and DNA was extracted and double-digested with $F s e I$ and Sbfl as described above. A genomic sequence

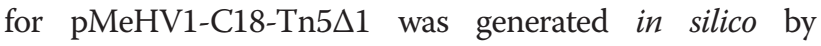


insertion of the Tn5 transposon sequence to simulate the $\mathrm{TR}_{\mathrm{S}}$ insertion genotype. Analogous genomic sequences were also generated for clones with the $\mathrm{IR}_{\mathrm{S}}$ insertion and the $\mathrm{IR}_{\mathrm{S}} / \mathrm{TR}_{\mathrm{S}}$ dual insertion genotypes. Selected clones representing the dual-icp4 deletion genotype were identified by restriction endonuclease digestion and subsequently transfected back into CEFs, and cultures were monitored for the development of CPE.

\section{Additional file}

Additional file 1: Supplemental Table S1. Details of transposon gene interruptions into the MeHV-1 infectious clone pMeHV1-C18. Supplemental Table S2: Oligonucleotides used in this study. Supplemental Figure S1: Primary chicken embryo fibroblasts (CEFs) were used as a negative control. (PDF $121 \mathrm{~kb}$ )

\section{Abbreviations}

BoHV-1: Bovine herpesvirus 1; CEFs: Chicken embryo fibroblasts; CPE: Cytopathic effect; EHV-1: Equine herpesvirus 1; eGFP: Enhanced green fluorescent protein; GaHV-2: Gallid herpesvirus 2; GaHV-3: Gallid herpesvirus 3; gC: Glycoprotein C; gD: Glycoprotein D; gE: Glycoprotein E; gK: Glycoprotein K; gM: Glycoprotein M; HHV-1: Human herpesvirus 1; HHV-3: Human herpesvirus 3; HVT: Turkey herpesvirus; iBAC: Infectious bacterial artificial chromosome; IRL: Internal repeat long; IRS: Internal repeat short; MD: Marek's disease; MeHV-1: Meleagrid herpesvirus 1; RE: Restriction enzyme; SuHV-1: Suid herpesvirus 1; TRL: Terminal repeat long; TRS: Terminal repeat short; UL: Unique long; US: Unique short.

\section{Competing interests}

The authors declare that they have no competing interests.

\section{Authors' contributions}

RNH participated in design of the study, carried out the molecular studies and drafted the manuscript. JM and EVF participated in design of the study and critical revision of the manuscript. TJM conceived the study, and participated in its design and coordination and assisted with drafting the manuscript. All authors read and approved the final manuscript.

\section{Acknowledgements}

This research was funded by Poultry CRC, established and supported under the Australian Government's Cooperative Research Centres Program. RNH was supported by an Australian Postgraduate Award and by the Poultry CRC. The authors wish to thank Michael Frese for critical revision of the manuscript.

\section{Author details}

'School of Veterinary Science, The University of Queensland, Gatton, QLD 4343, Australia. ${ }^{2}$ Poultry CRC, University of New England, Armidale, NSW 2351, Australia. ${ }^{3}$ Present address: Commonwealth Scientific and Industrial Research Organisation - Biosecurity Flagship, Black Mountain 2601, Australia. ${ }^{4}$ Animal Science, Department of Agriculture, Fisheries and Forestry, Brisbane, QLD 4072, Australia. ${ }^{5}$ Queensland Alliance for Agriculture and Food Innovation, Centre for Animal Science, The University of Queensland, Brisbane, QLD 4072, Australia.

Received: 26 June 2015 Accepted: 17 August 2015

Published online: 27 August 2015

\section{References}

1. Kawamura H, King Jr DJ, Anderson DP. A herpesvirus isolated from kidney cell culture of normal turkeys. Avian Dis. 1969;13:853-63.

2. Witter RL, Nazerian K, Purchase HG, Burgoyne GH. Isolation from turkeys of a cell-associated herpesvirus antigenically related to Marek's disease virus. Adv Virus Res. 1970;31:525-38.

3. Okazaki W, Purchase HG, Burmester BR. Protection against Marek's disease by vaccination with a herpesvirus of turkeys. Avian Dis. 1970;14:413-29.
4. Eidson CS, Ellis MN, Kleven SH. Reduced vaccinal protection of turkey herpesvirus against field strains of Marek's disease herpesvirus. Poult Sci. 1981;60:317-22.

5. Witter RL. Increased virulence of Marek's disease virus field isolates. Avian Dis. 1997;41:149-63.

6. Palya V, Kiss I, Tatar-Kis T, Mato T, Felfoldi B, Gardin Y. Advancement in vaccination against Newcastle disease: recombinant HVT NDV provides high clinical protection and reduces challenge virus shedding with the absence of vaccine reactions. Avian Dis. 2012;56:282-7.

7. Le Gros FX, Dancer A, Giacomini C, Pizzoni L, Bublot M, Graziani M, et al. Field efficacy trial of a novel HVT-IBD vector vaccine for 1-day-old broilers. Vaccine. 2009;27:592-6.

8. Johnson DI, Vagnozzi A, Dorea F, Riblet SM, Mundt A, Zavala G, et al. Protection against infectious laryngotracheitis by in ovo vaccination with commercially available viral vector recombinant vaccines. Avian Dis. 2010;54:1251-9.

9. Rauw F, Palya V, Van Borm S, Welby S, Tatar-Kis T, Gardin Y, et al. Further evidence of antigenic drift and protective efficacy afforded by a recombinant $\mathrm{HVT}-\mathrm{H} 5$ vaccine against challenge with two antigenically divergent Egyptian clade 2.2.1 HPAI H5N1 strains. Vaccine. 2011;29:2590-600.

10. Gao H, Cui H, Cui $X$, Shi $X$, Zhao $Y$, Zhao $X$, et al. Expression of HA of HPAl $\mathrm{H} 5 \mathrm{~N} 1$ virus at US2 gene insertion site of turkey herpesvirus induced better protection than that at US10 gene insertion site. PLoS One. 2011;6, e22549.

11. Afonso CL, Tulman ER, Lu Z, Zsak L, Rock DL, Kutish GF. The genome of turkey herpesvirus. J Virol. 2001;75:971-8.

12. Kingham BF, Zelník V, Kopáček J, Majerciak V, Ney E, Schmidt CJ. The genome of herpesvirus of turkeys: comparative analysis with Marek's disease viruses. J Gen Virol. 2001;82:1123-35.

13. Spatz SJ, Silva RF. Polymorphisms in the repeat long regions of oncogenic and attenuated pathotypes of Marek's disease virus 1. Virus Genes. 2007;35:41-53

14. Fields BN, Knipe DM, Howley PM. Fields' virology. 5th ed. Philadelphia: Wolters Kluwer/Lippincott Williams \& Wilkins; 2007.

15. Weber $P$, Levine M, Glorioso J. Rapid identification of nonessential genes of herpes simplex virus type 1 by Tn5 mutagenesis. Science. 1987;236:576-9.

16. Brune W, Menard C, Hobom U, Odenbreit S, Messerle M, Koszinowski UH. Rapid identification of essential and nonessential herpesvirus genes by direct transposon mutagenesis. Nat Biotechnol. 1999;17:360-4.

17. Smith GA, Enquist LW. Construction and transposon mutagenesis in Escherichia coli of a full-length infectious clone of pseudorabies virus, an alphaherpesvirus. J Virol. 1999;73:6405-14.

18. Chattoo JP, Stevens MP, Nair V. Rapid identification of non-essential genes for in vitro replication of Marek's disease virus by random transposon mutagenesis. J Virol Methods. 2006;135:288-91.

19. Robinson KE, Meers J, Gravel JL, MCCarthy FM, Mahony TJ. The essential and non-essential genes of bovine herpesvirus 1. J Gen Virol. 2008;89:2851-63.

20. Mahony TJ, Hall RN, Walkden-Brown S, Meers J, Gravel JL, West L, et al. Genomic deletions and mutations resulting in the loss of eight genes reduce the in vivo replication capacity of Meleagrid herpesvirus 1. Virus Genes. 2015;51:85-95.

21. Hall RN, Meers J, Mitter N, Fowler EV, Mahony TJ. The meleagrid herpesvirus 1 genome is partially resistant to transposition. Avian Dis. 2013:57:380-6.

22. Borst EM, Hahn G, Koszinowski UH, Messerle M. Cloning of the human cytomegalovirus (HCMV) genome as an infectious bacterial artificial chromosome in Escherichia coli: a new approach for construction of HCMV mutants. J Virol. 1999;73:8320-9.

23. Hearn C, Preeyanon L, Hunt HD, York IA. An MHC class I immune evasion gene of Mareks disease virus. Virology. 2015;475:88-95.

24. Isfort $\mathrm{R}$, Jones $\mathrm{D}$, Kost $\mathrm{R}$, Witter $\mathrm{R}$, Kung $\mathrm{HJ}$. Retrovirus insertion into herpesvirus in vitro and in vivo. Proc Natl Acad Sci U S A. 1992;89:991-5.

25. Tischer BK, Schumacher D, Messerle M, Wagner M, Osterrieder N. The products of the UL10 (gM) and the UL49.5 genes of Marek's disease virus serotype 1 are essential for virus growth in cultured cells. J Gen Virol. 2002;83:997-1003.

26. Zhang Z, Selariu A, Warden C, Huang G, Huang Y, Zaccheus O, et al. Genome-wide mutagenesis reveals that ORF7 is a novel VZV skin-tropic factor. PLoS Pathog. 2010;6, e1000971.

27. Dijkstra JM, Visser N, Mettenleiter TC, Klupp BG. Identification and characterization of pseudorabies virus glycoprotein $\mathrm{gM}$ as a nonessential virion component. J Virol. 1996;70:5684-8. 
28. Fuchs W, Mettenleiter TC. DNA sequence of the UL6 to UL20 genes of infectious laryngotracheitis virus and characterization of the UL10 gene product as a nonglycosylated and nonessential virion protein. J Gen Virol. 1999:80:2173-82.

29. Seyboldt C, Granzow H, Osterrieder N. Equine herpesvirus 1 (EHV-1) glycoprotein M: effect of deletions of transmembrane domains. Virology. 2000;278:477-89.

30. Baines JD, Roizman B. The open reading frames UL3, UL4, UL10, and UL16 are dispensable for the replication of herpes simplex virus 1 in cell culture. J Virol. 1991;65:938-44

31. Yachida S, Kondo T, Hirai K, Izawa H, Mikami T. Establishment of a variant type of turkey herpesvirus which releases cell-free virus into the culture medium in large quantities. Arch Virol. 1986;91:183-92.

32. Le Sage $V$, Jung M, Alter JD, Wills EG, Johnston SM, Kawaguchi Y, et al. The herpes simplex virus type 2 UL21 protein is essential for virus propagation. J Virol. 2013;87:5904-15.

33. Takakuwa H, Goshima F, Koshizuka T, Murata T, Daikoku T, Nishiyama Y. Herpes simplex virus encodes a virion-associated protein which promotes long cellular processes in over-expressing cells. Genes Cells. 2001;6:955-66.

34. de Wind N, Wagenaar F, Pol J, Kimman T, Berns A. The pseudorabies virus homology of the herpes simplex virus UL21 gene product is a capsid protein which is involved in capsid maturation. J Virol. 1992;66:7096-103.

35. Baines JD, Koyama AH, Huang T, Roizman B. The UL21 gene products of herpes simplex virus 1 are dispensable for growth in cultured cells. J Virol. 1994;68:2929-36.

36. Muto Y, Goshima F, Ushijima Y, Kimura H, Nishiyama Y. Generation and characterization of UL21-null herpes simplex virus type 1. Front Microbiol. 2012:3:394.

37. Hansen $\mathrm{K}$, Napier I, Koen M, Bradford S, Messerle M, Bell E, et al. In vitro transposon mutagenesis of an equine herpesvirus 1 genome cloned as a bacterial artificial chromosome. Arch Virol. 2006;151:2389-405.

38. Weinheimer SP, Boyd BA, Durham SK, Resnick JL, O'Boyle 2nd DR. Deletion of the VP16 open reading frame of herpes simplex virus type 1. J Virol. 1992;66:258-69.

39. Yanagida N, Yoshida S, Nazerian K, Lee LF. Nucleotide and predicted amino acid sequences of Marek's disease virus homologues of herpes simplex virus major tegument proteins. J Gen Virol. 1993;74:1837-45.

40. Kopáček J, Zelník V, Koptidesová D, Pastoreková S, Pastorek J, Brasseur R, et al. Herpesvirus of turkeys homologue of HSV VP16 is structurally related to varicella zoster virus trans-inducing protein encoded by ORF10. Virus Genes. 1997:15:45-52

41. Cohen Jl, Seidel K. Varicella-zoster virus (VZV) open reading frame 10 protein, the homolog of the essential herpes simplex virus protein VP16, is dispensable for VZV replication in vitro. J Virol. 1994;68:7850-8.

42. Dorange F, Tischer BK, Vautherot J-F, Osterrieder N. Characterization of Marek's disease virus serotype 1 (MDV-1) deletion mutants that lack UL46 to UL49 genes: MDV-1 UL49, encoding VP22, is indispensable for virus growth. J Virol. 2002;76:1959-70.

43. Fuchs W, Granzow H, Klupp BG, Kopp M, Mettenleiter TC. The UL48 tegument protein of pseudorabies virus is critical for intracytoplasmic assembly of infectious virions. J Virol. 2002;76:6729-42.

44. von Einem J, Schumacher D, O'Callaghan DJ, Osterrieder N. The alpha-TIF (VP16) homologue (ETIF) of equine herpesvirus 1 is essential for secondary envelopment and virus egress. J Virol. 2006;80:2609-20.

45. Che X, Reichelt M, Sommer MH, Rajamani J, Zerboni L, Arvin AM. Functions of the ORF9-to-ORF12 gene cluster in varicella-zoster virus replication and in the pathogenesis of skin infection. J Virol. 2008;82:5825-34.

46. Hutchinson L, Goldsmith K, Snoddy D, Ghosh H, Graham FL, Johnson DC. Identification and characterization of a novel herpes simplex virus glycoprotein, gK, involved in cell fusion. J Virol. 1992;66:5603-9.

47. Hutchinson L, Johnson DC. Herpes simplex virus glycoprotein $\mathrm{K}$ promotes egress of virus particles. J Virol. 1995;69:5401-13.

48. Klupp BG, Baumeister J, Dietz P, Granzow H, Mettenleiter TC. Pseudorabies virus glycoprotein $\mathrm{gK}$ is a virion structural component involved in virus release but is not required for entry. J Virol. 1998;72:1949-58.

49. Osterrieder K, Vautherot J-F. The genome content of Marek's disease-like viruses. In: Davison F, Nair V, editors. In Marek's Disease: An Evolving Problem. Oxford: Academic; 2004. p. 17-31.

50. Jons A, Dijkstra JM, Mettenleiter TC. Glycoproteins M and N of pseudorabies virus form a disulfide-linked complex. J Virol. 1998;72:550-7.

51. Jayachandra S, Baghian A, Kousoulas KG. Herpes simplex virus type 1 glycoprotein $\mathrm{K}$ is not essential for infectious virus production in actively replicating cells but is required for efficient envelopment and translocation of infectious virions from the cytoplasm to the extracellular space. J Virol. 1997;71:5012-24

52. Neubauer A, Osterrieder N. Equine herpesvirus type 1 (EHV-1) glycoprotein $K$ is required for efficient cell-to-cell spread and virus egress. Virology. 2004;329:18-32.

53. Tischer BK, Schumacher D, Chabanne-Vautherot D, Zelník V, Vautherot JF, Osterrieder N. High-level expression of Marek's disease virus glycoprotein C is detrimental to virus growth in vitro. J Virol. 2005;79:5889-99.

54. Jarosinski KW, Osterrieder N. Further analysis of Marek's disease virus horizontal transmission confirms that $\mathrm{U}(\mathrm{L}) 44(\mathrm{gC})$ and $\mathrm{U}(\mathrm{L}) 13$ protein kinase activity are essential, while $U(S) 2$ is nonessential. J Virol. 2010;84:7911-6.

55. Zhang Z, Schwartz S, Wagner L, Miller W. A greedy algorithm for aligning DNA sequences. J Comput Biol. 2000;7:203-14.

56. Morgan RW, Cantello JL, McDermott CH. Transfection of chicken embryo fibroblasts with Marek's disease virus DNA. Avian Dis. 1990:34:345-51.

57. Isfort RJ, Qian Z, Jones D, Silva RF, Witter R, Kung HJ. Integration of multiple chicken retroviruses into multiple chicken herpesviruses: herpesviral gD as a common target of integration. Virology. 1994;203:125-33.

58. Darteil R, Bublot M, Laplace E, Bouquet JF, Audonnet JC, Riviere M. Herpesvirus of turkey recombinant viruses expressing infectious bursal disease virus (IBDV) VP2 immunogen induce protection against an IBDV virulent challenge in chickens. Virology. 1995;211:481-90.

59. Baigent SJ, Petherbridge $\amalg$, Smith LP, Zhao Y, Chesters PM, Nair VK. Herpesvirus of turkey reconstituted from bacterial artificial chromosome clones induces protection against Marek's disease. J Gen Virol. 2006:87:769-76.

60. Sakaguchi M, Urakawa T, Hirayama Y, Miki N, Yamamoto M, Zhu GS, et al. Marek's disease virus protein kinase gene identified within the short unique region of the viral genome is not essential for viral replication in cell culture and vaccine-induced immunity in chickens. Virology. 1993;195:140-8.

61. Anderson AS, Parcells MS, Morgan RW. The glycoprotein D (US6) homolog is not essential for oncogenicity or horizontal transmission of Marek's disease virus. J Virol. 1998;72:2548-53.

62. Schumacher D, Tischer BK, Fuchs W, Osterrieder N. Reconstitution of Marek's disease virus serotype 1 (MDV-1) from DNA cloned as a bacterial artificial chromosome and characterization of a glycoprotein B-negative MDV-1 mutant. J Virol. 2000;74:11088-98.

63. Schumacher D, Tischer BK, Reddy SM, Osterrieder N. Glycoproteins E and I of Marek's disease virus serotype 1 are essential for virus growth in cultured cells. J Virol. 2001;75:11307-18.

64. Kamil JP, Tischer BK, Trapp S, Nair VK, Osterrieder N, Kung HJ. VLIP, a vira lipase homologue, is a virulence factor of Marek's disease virus. J Virol. 2005;79:6984-96.

65. Blondeau C, Chbab N, Beaumont C, Courvoisier K, Osterrieder N, Vautherot JF, et al. A full UL13 open reading frame in Marek's disease virus (MDV) is dispensable for tumor formation and feather follicle tropism and cannot restore horizontal virus transmission of rRB-1B in vivo. Vet Res. 2007;38:419-33.

66. Chbab N, Chabanne-Vautherot D, Francineau A, Osterrieder N, Denesvre C, Vautherot JF. The Marek's disease virus (MDV) protein encoded by the UL17 ortholog is essential for virus growth. Vet Res. 2009;40:28.

67. Sun A, Lee LF, Khan OA, Heidari M, Zhang H, Lupiani B, et al. Deletion of Marek's disease virus large subunit of ribonucleotide reductase impairs virus growth in vitro and in vivo. Avian Dis. 2013:57:464-8.

68. Goldstein DJ, Weller SK. Herpes simplex virus type 1-induced ribonucleotide reductase activity is dispensable for virus growth and DNA synthesis: isolation and characterization of an ICP6 lacZ insertion mutant. J Virol. 1988;62:196-205.

69. Ligas MW, Johnson DC. A herpes simplex virus mutant in which glycoprotein D sequences are replaced by beta-galactosidase sequences binds to but is unable to penetrate into cells. J Virol. 1988;62:1486-94.

70. Malik AK, Martinez R, Muncy L, Carmichael EP, Weller SK. Genetic analysis of the herpes simplex virus type 1 UL 9 gene: isolation of a LacZ insertion mutant and expression in eukaryotic cells. Virology. 1992;190:702-15.

71. Purves FC, Spector D, Roizman B. UL34, the target of the herpes simplex virus $U(S) 3$ protein kinase, is a membrane protein which in its unphosphorylated state associates with novel phosphoproteins. J Virol. 1992;66:4295-303. 
72. Coulter LJ, Moss HW, Lang J, McGeoch DJ. A mutant of herpes simplex virus type 1 in which the UL13 protein kinase gene is disrupted. J Gen Virol. 1993;74(Pt 3):387-95.

73. Balan P, Davis-Poynter N, Bell S, Atkinson H, Browne H, Minson T. An analysis of the in vitro and in vivo phenotypes of mutants of herpes simplex virus type 1 lacking glycoproteins gG, gE, gl or the putative gJ. J Gen Virol. 1994;75:1245-58.

74. Martin DW, Weber PC. The a sequence is dispensable for isomerization of the herpes simplex virus type 1 genome. J Virol. 1996;70:8801-12.

75. Salmon B, Cunningham C, Davison AJ, Harris WJ, Baines JD. The herpes simplex virus type $1 \mathrm{U}(\mathrm{L}) 17$ gene encodes virion tegument proteins that are required for cleavage and packaging of viral DNA. J Virol. 1998;72:3779-88.

76. Desai $P$, Sexton GL, McCaffery JM, Person S. A null mutation in the gene encoding the herpes simplex virus type 1 UL37 polypeptide abrogates virus maturation. J Virol. 2001;75:10259-71.

77. Mallory S, Sommer M, Arvin AM. Analysis of the glycoproteins I and E of varicella-zoster virus (VZV) using deletional mutations of VZV cosmids. J Infect Dis. 1998;178:22-6.

78. Sato $B$, Ito $H$, Hinchliffe $S$, Sommer MH, Zerboni L, Arvin AM. Mutational analysis of open reading frames 62 and 71, encoding the varicella-zoster virus immediate-early transactivating protein, IE62, and effects on replication in vitro and in skin xenografts in the SCID-hu mouse in vivo. J Virol. 2003;77:5607-20.

79. de Wind N, Zijderveld A, Glazenburg K, Gielkens A, Berns A. Linker insertion mutagenesis of herpesviruses: inactivation of single genes within the Us region of pseudorabies virus. J Virol. 1990;64:4691-6.

80. Hanssens FP, Nauwynck HJ, Mettenleiter TC. Role of glycoprotein gD in the adhesion of pseudorabies virus infected cells and subsequent cell-associated virus spread. Arch Virol. 1995;140:1855-62.

81. Kopp M, Klupp BG, Granzow H, Fuchs W, Mettenleiter TC. Identification and characterization of the pseudorabies virus tegument proteins UL46 and UL47: role for UL47 in virion morphogenesis in the cytoplasm. J Virol. 2002;76:8820-33.

82. Klupp BG, Granzow H, Karger A, Mettenleiter TC. Identification, subviral localization, and functional characterization of the pseudorabies virus UL17 protein. J Virol. 2005;79:13442-53.

83. Furth JJ, Whitbeck JC, Lawrence WC, Bello L. Construction of a viable BHV1 mutant lacking most of the short unique region. Arch Virol. 1997;142:2373-87.

\section{Submit your next manuscript to BioMed Central and take full advantage of:}

- Convenient online submission

- Thorough peer review

- No space constraints or color figure charges

- Immediate publication on acceptance

- Inclusion in PubMed, CAS, Scopus and Google Scholar

- Research which is freely available for redistribution 